\title{
Fuzzy Stabilizer in IMTL-Algebras
}

\author{
Maosen Xie* \\ Institute of Mathematics and Finance, Sichuan University of Arts and Science, Dazhou, Sichuan, 635000, China
}

Received: 16 Sep. 2013, Revised: 14 Dec. 2013, Accepted: 15 Dec. 2013

Published online: 1 Sep. 2014

\begin{abstract}
In this paper, we introduced two types of fuzzy stabilizers $S t_{R}^{\otimes}(F)$ and $S t_{R}^{\oplus}(F)$ of a fuzzy set $F$ and the stabilizer $S t^{\vee}(F, G)$ of $F$ with respect to $G$ in IMTL-algebras. We proved that $S t_{R}^{\otimes}(F), S t_{R}^{\oplus}(F)$ and $S t^{\vee}(F, G)$ are fuzzy filters of $M$. We investigated some properties of the stabilizers. By introducing the $\widetilde{\otimes}$ operation of two fuzzy filters, we prove that $\widetilde{\otimes}$ and $S t^{\vee}(\cdot, \cdot)$ form an adjoint pair and the set of all fuzzy filters $\mathscr{F}(M)$ is a residuated lattice.
\end{abstract}

Keywords: IMTL-algebra, Fuzzy filter, Fuzzy stablizer.

\section{Introduction}

Algebraic method plays a key role in studying fuzzy logic, and any logical algebra systems corresponding to various logical systems are established accordingly [1,2]. MTL-algebras are the corresponding algebraic structures of Esteva and Godo's monoidal $t$-norm based logic (MTL). IMTL-algebras, which are the algebra structures for monoidal $t$-norm based logic with an involutive negation (IMTL), are the important sub-classes of MTLalgebras. The main examples of IMTL-algebras are the real unit interval $[0,1]$ endowed with the structure induced by a left-continuous $t$-norm with an involutive negation and the Lindenbaum algebra of the IMTL (the quotient of the free algebra of formulas with respect to the logical equivalence). MV-algebras and NM-algebras, which is called $R_{0}$-algebras in [6], are the most known classes of IMTL-algebras. For more details of these algebras, we refer the reader to [3,4,5,6,7,8,9].

Up to now, these relevant logical algebra structures have already been widely studied. In particular, emphasis seems to have been put on filters theory. Hájek [3] proved the completeness of basic logical system BL. Turunnen $[10,11]$ studied some properties of the filters and prime filters of BL-algebras (he called them deductive systems and prime deductive systems, respectively). In [12], he introduced the notion of Boolean filters (Boolean deductive systems) in BL-algebras and derived some characterizations of Boolean filters. Xu, Qin and Jun [13, $14,15,16]$ proposed the notions of positive implicative filter and fuzzy positive implicative filter, and derived several characterizations of fuzzy positive implicative filters in lattice implication algebras. Liu and $\mathrm{Li}[17,18]$ proposed the notions of fuzzy Boolean filters and fuzzy positive implicative filters of BL-algebras and $R_{0}$-algebras. Some properties of fuzzy Boolean filters and fuzzy positive implicative filters of BL-algebras and $R_{0}$-algebras are derived. Recently, Zhang and Zhou [19] proved that the filters set of a residuated lattice forms a residuated lattice and the prime Boolean filters set is a quasi-Boolean algebra by introducing some operations among filters.

In the present paper, we studied two types of fuzzy stabilizers $S t_{R}^{\otimes}(F)$ and $S t_{R}^{\oplus}(F)$ of a fuzzy set $F$ and the fuzzy stabilizer $S t^{\vee}(F, G)$ of $F$ with respect to $G$, for fuzzy subsets $F$ and $G$ in IMTL-algebras. We investigated some properties of the fuzzy stabilizers and show that $S t_{R}^{\otimes}(F), S t_{R}^{\oplus}(F)$ and $S t^{\vee}(F, G)$ are fuzzy filters of $M$. By introducing the $\widetilde{\otimes}$ operation of two fuzzy filters, we prove that $\widetilde{\otimes}$ and $S t^{\vee}(\cdot, \cdot)$ is an adjoint pair and the set $\mathscr{F}(M)$ of all fuzzy filters forms a residuated lattice.

\section{Preliminaries}

Throughout this paper, let $M$ denote an IMTL-algebra. Here we recall some definitions and results about IMTLalgebra which will be needed in the following.

Definition 2.1 (see [4]). A MTL-algebra is a bounded residuated lattice $(M, \vee, \wedge, \otimes, \rightarrow, 0,1)$, where $\vee$ and $\wedge$ are the lattice meet and join operations and $(\otimes, \rightarrow)$ is a

\footnotetext{
*Corresponding author e-mail: dazouxms@163.com
} 
residuated pair, satisfying the pre-linearity equation

$$
(x \rightarrow y) \vee(y \rightarrow x)=1 .
$$

An IMTL-algebra is a MTL-algebra satisfying

$$
\neg \neg x=x,
$$

where $\neg x=x \rightarrow 0$.

Let $M$ be an IMTL-algebra. For any $x, y \in M$, define $x \oplus y=\neg x \rightarrow y$. It is easy to verify that $\oplus$ are commutative, associative and $x \oplus y=\neg(\neg x \otimes \neg y)$.

Example 2.2. Let $[0,1]$ be the real unit interval. $\otimes$ is a left-continuous $t$-norm with an order-reversing involution and $\rightarrow$ is its residuum, then $([0,1], \otimes, \rightarrow)$ is an IMTLalgebra called the IMTL-unit interval.

Lemma 2.3 (see $[4,7]$ ). Let $M$ be an IMTL-algebra. Then for any $x, y, z \in M$,

(P1) $(M, \otimes, 1)$ is a commutative semigroup with the unit element 1 ,

(P2) $\oplus$ is a monotone operator,

(P3) $x \otimes y \leq x \wedge y \leq x \vee y \leq x \oplus y$,

(P4) $x \otimes y \leq z$ if and only if $x \leq y \rightarrow z$,

(P5) $x \otimes y \rightarrow z=x \rightarrow(y \rightarrow z), x \rightarrow(y \rightarrow x \otimes y)=1$,

(P6) $x \rightarrow y \leq x \vee z \rightarrow y \vee z, x \rightarrow y \leq x \otimes z \rightarrow y \otimes z$,

(P7) $x \otimes(x \rightarrow y) \leq y, x \leq y \rightarrow x \otimes y$,

(P8) $x \otimes \neg x=0, x \oplus \neg x=1$,

(P9) $x \otimes(y \vee z)=(x \otimes y) \vee(x \otimes z)$,

(P10) $x \vee(y \otimes z) \geq(x \vee y) \otimes(x \vee z)$,

(P11) $(x \leftrightarrow y) \otimes(y \leftrightarrow z) \leq x \leftrightarrow z$, where $x \leftrightarrow y=(x \rightarrow$ y) $\wedge(y \rightarrow x)$,

(P12) $(x \leftrightarrow y) \otimes(z \leftrightarrow u) \leq(x \triangle z) \leftrightarrow(y \triangle u)$, where $\triangle \in\{\wedge, \vee, \otimes, \rightarrow\}$.

A subset $A$ of $M$ is called a filter of $M$ if $(i) 1 \in A$, (ii) $x \in A$ and $x \leq y$ imply $y \in A$, (iii) $x, y \in A$ implies $x \otimes$ $y \in A$. It is easy to prove that a subset $A$ is a filter if and only if $A$ satisfies $(i)$ and $(i v) x, x \rightarrow y \in A$ imply $y \in A$. A filter $A$ is called prime if $(v) \forall a, b \in M, x \vee y \in A$ implies $a \in A$ or $b \in A[6,12]$.

Definition 2.4 (see [19]). A fuzzy set $F$ of $M$ is called a fuzzy filter of $M$ if it satisfies:

(F1) $F(1) \geq F(x)$, for any $x \in M$,

(F2) if $x \leq y$ then $F(x) \leq F(y)$,

(F3) $F(x \otimes y) \geq F(x) \wedge F(y)$, for any $x, y \in M$.

The empty set $\emptyset$ is also viewed as a fuzzy filter of $M$, and the set of all fuzzy filters of $\mathrm{M}$ is denoted by $\mathscr{F}(M)$.

Proposition 2.5 (see [19]). A fuzzy set $F$ of $M$ is a fuzzy filter if and only if $F$ satisfies $(F 1)$ and

$(F 4) F(y) \geq F(x) \wedge F(x \rightarrow y)$ for any $x, y \in M$.

Definition 2.6 (see [19]). Let $F$ be a fuzzy filter of $M$. $F$ is called a fuzzy prime filter if $F(x \vee y)=F(x) \vee F(y)$ for any $x, y \in M$.

Proposition 2.7 (see [19]). A fuzzy set $F$ of $M$ is a fuzzy prime Boolean filter if and only if $F(x \oplus y)=F(x) \vee$ $F(y)$ for any $x, y \in M$.

Definition 2.8 (see [19]). Let $G$ be a fuzzy set of $M$. The fuzzy filter generated by $G$ is defined as

$$
\langle G\rangle=\bigcap_{\substack{F \in \mathscr{F}(M) \\ G \subseteq F}} F
$$

Lemma 2.9 (see [19]). Let $G$ be a nonempty fuzzy set of $M$. Then for $x \in M$,

$$
\langle G\rangle(x)=\bigvee\left\{\bigwedge_{k=1}^{n} G\left(a_{k}\right) \mid a_{1}, \cdots, a_{n} \in M, x \geq a_{1} \otimes \cdots \otimes a_{n}\right\} .
$$

Definition 2.10 (see [19]). A fuzzy subset $R$ on $M \times M$ is called a fuzzy congruence relation if $R$ is a fuzzy equivalent relation and satisfies

$$
R(x \triangle z, y \triangle u) \geq R(x, y) \wedge R(z, u),
$$

for all $x, y, z, u \in M$, where $\triangle \in\{\wedge, \vee, \otimes, \rightarrow\}$.

Proposition 2.11 (see [19]). Let $R$ be a fuzzy congruence relation on $M$. Then $R(x, y)=R(x \leftrightarrow y, 1)$ for all $x, y \in M$

Proposition 2.12 (see [19]). Let $R$ be a fuzzy congruence relation on $M$. Then $R(x, \cdot)$ is a fuzzy filter for every $x \in M$.

Proposition 2.13 (see [19]). Let $F$ be a fuzzy filter of IMTL-algebra $M$ and $F(1)=1$. By means of $F$ we can generate a fuzzy congruence relation $R(x, y)=$ $F((x \rightarrow y) \wedge(y \rightarrow x))$ on $M \times M$.

Proposition 2.14. For a fuzzy congruence relation $R$ we have $R(x, y)=R(\neg x, \neg y)$ for all $x, y \in M$.

Proof. On one hand, $R(\neg x, \neg y)=R(x t o 0, y \rightarrow 0) \geq$ $R(x, y) \wedge R(0,0)=R(x, y)$. On the other hand, $R(x, y)=$ $R((x \rightarrow 0) \rightarrow 0,(y \rightarrow 0) \rightarrow 0) \geq R(x \rightarrow 0, y \rightarrow$ $0) \wedge R(0,0)=R(\neg x, \neg y)$. Hence $R(x, y)=R(\neg x, \neg y)$.

\section{Fuzzy stabilizer with respect to a fuzzy congruence relation $R$ in IMTL-algebras}

Definition 3.1. Let $F$ be a fuzzy set of $M$ and $R$ be a fuzzy congruence relation on $M$. The fuzzy $\otimes$-stabilizer and fuzzy $\oplus$-stabilizer of $F$ with respect to $R$ are defined by:

$$
\begin{aligned}
S t_{R}^{\otimes}(F)(x) & =\bigwedge_{z \in M}\{F(z) \rightarrow R(z, z \otimes x)\}, \\
S t_{R}^{\oplus}(F)(x) & =\bigwedge_{z \in M}\{F(z) \rightarrow R(z \oplus \neg x, z)\}, x \in M,
\end{aligned}
$$

where $\rightarrow$ is the residuum implication with respect to a leftcontinuous $t$-norm $\otimes$ with an order-reversing involution on $[0,1]$.

Remark 3.2. (1) Let $R$ be an identity relation on $M$. Then for any $x \in M$,

$$
\begin{aligned}
& S t_{I d}^{\otimes}(F)(x)=\bigwedge_{z \in M}\{F(z) \rightarrow I d(z, z \otimes x)\}=\bigvee_{z \neq z \otimes x} F(z) \rightarrow 0, \\
& S t_{I d}^{\oplus}(F)(x)=\bigwedge_{z \in M}\{F(z) \rightarrow I d(z \oplus \neg x, z)\}=\bigvee_{z \neq z \oplus \neg x} F(z) \rightarrow 0 .
\end{aligned}
$$

(2) If $R$ is an identity relation $I d$ on $M$ and $X$ is a classic subset of $M$, i.e., $F=\chi_{X}$. Then 
$S t_{I d}^{\otimes}(X)=\{x \in M \mid \forall z \in X, z \otimes x=z\}=\{x \in M \mid \forall z \in$ $X, x \rightarrow \neg z=\neg z\}$,

$S t_{I d}^{\oplus}(X)=\{x \in M \mid \forall z \in X, z \oplus \neg X=z\}=\{x \in M \mid \forall z \in$ $X, x \rightarrow z=z\}$.

The above equations mean that $z \otimes x$ identity with $z$ and $z \oplus \neg x$ identity with $z$ for every element $z \in X$. From logical point of view, if $X$ is a formula set then $S t_{I d}^{\oplus}(X)$ corresponds to the set of formulae that every formula in $X$ that implies $z$ equals to $z, S t_{I d}^{\otimes}(X)$ corresponds to the set of formulaes that every formula in $X$ that implies $\neg z$ equals to $\neg z$ for any formula $z \in X$. This means that the formulas in $S t_{I d}^{\otimes}(X)$ and $S t_{I d}^{\oplus}(X)$ have some stability for implication operation. Hence $S t_{I d}^{\otimes}(X)$ and $S t_{I d}^{\oplus}(X)$ may be called $\otimes$-stabilizer and $\oplus$-stabilizer of $X$ respectively. Further $S t_{R}^{\otimes}(F)$ and $S t_{R}^{\oplus}(F)$ are called fuzzy $\otimes$-stabilizer and fuzzy $\oplus$-stabilizer of fuzzy set $F$ respectively.

Example 3.3. Let $M=\{0, a, b, c, d, 1\}$ be a set with Fig.1 as a partial order. Define two binary operations " $\otimes$ " and " $\rightarrow$ " on $M$ as Table 1 and Table 2 respectively:

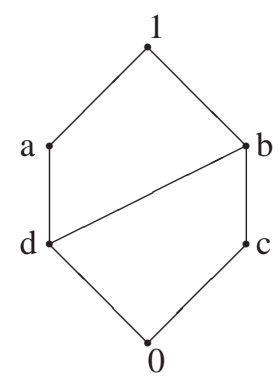

Fig. 1

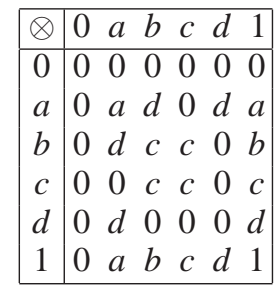

Table 1

\begin{tabular}{|c|cccccc|}
\hline$\rightarrow$ & 0 & $a$ & $b$ & $c$ & $d$ & 1 \\
\hline 0 & 1 & 1 & 1 & 1 & 1 & 1 \\
$a$ & $c$ & 1 & $b$ & $c$ & $b$ & 1 \\
$b$ & $d$ & $a$ & 1 & $b$ & $a$ & 1 \\
$c$ & $a$ & $a$ & 1 & 1 & $a$ & 1 \\
$d$ & $b$ & 1 & 1 & $b$ & 1 & 1 \\
1 & 0 & $a$ & $b$ & $c$ & $d$ & 1 \\
\hline
\end{tabular}

Table 2

Then $M$ is an IMTL-algebra. Define a fuzzy set $F$ of $M$ by

$$
F(x)=\left\{\begin{array}{l}
1, \text { for } x=1, \\
0.7, \text { for } x \in\{b, c\}, \\
0.3, \text { for } x \in\{0, a, d\} .
\end{array}\right.
$$

The fuzzy congruence relation $R$ is generated by $F$. Then fuzzy $\otimes$-stabilizer and fuzzy $\oplus$-stabilizer of $F$ are

$$
\begin{aligned}
& S t_{R}^{\otimes}(F)=\left\{\begin{array}{l}
0.9, \text { for } x \in\{b, c, 1\}, \\
0.4, \text { for } x \in\{0, a, d\} .
\end{array}\right. \\
& S t_{R}^{\oplus}(F)=\left\{\begin{array}{l}
0.9, \text { for } x \in\{b, c, 1\}, \\
0.4, \text { for } x \in\{0, a, d\} .
\end{array}\right.
\end{aligned}
$$

In the following, we give some characterizations of fuzzy prime Boolean filters.

Theorem 3.4. Let $R$ be a fuzzy congruence relation and $F, G$ be two fuzzy sets of $M$. Then

(1) Denote $\chi_{\{1\}}(x)=\left\{\begin{array}{l}1, \text { for } x=1 \\ 0, \text { otherwise }\end{array} \quad\right.$ then $S t_{R}^{\otimes}\left(\chi_{\{1\}}\right)=R(1, \cdot)$;

(2) Denote $\chi_{\{0\}}(x)=\left\{\begin{array}{l}1, \text { for } x=0 \\ 0, \text { otherwise }\end{array} \quad\right.$ then $S t_{R}^{\otimes}\left(\chi_{\{0\}}\right)=M$;

(3) If $F \subseteq G$, then $S t_{R}^{\otimes}(G) \subseteq S t_{R}^{\otimes}(F)$ and $S t_{R}^{\oplus}(G) \subseteq$ $S t_{R}^{\oplus}(F)$.

(4) $S t_{R}^{\otimes}(F \cup G)=S t_{R}^{\otimes}(F) \cap S t_{R}^{\otimes}(G), \quad S t_{R}^{\oplus}(F \cup G)=$ $S t_{R}^{\oplus}(F) \cap S t_{R}^{\oplus}(G)$.

(5) $S t_{R}^{\otimes}(F \cap G)=S t_{R}^{\otimes}(F) \cup S t_{R}^{\otimes}(G), \quad S t_{R}^{\oplus}(F \cap G)=$ $S t_{R}^{\oplus}(F) \cup S t_{R}^{\oplus}(G)$.

(6) If we define a fuzzy set $\widetilde{F}: \widetilde{F}(x)=F(\neg x), x \in M$, then $S t_{R}^{\oplus}(\widetilde{F})=S t_{R}^{\otimes}(F)$.

Proof. (1) $S t_{R}^{\otimes}\left(\chi_{\{1\}}\right)(x)=\bigwedge_{z \in M}\left\{\chi_{\{1\}}(z) \rightarrow R(z, z \otimes x)\right\}$ $=1 \rightarrow R(1,1 \otimes x)=R(1, x)$. Hence $S t_{R}^{\otimes}\left(\chi_{\{1\}}\right)=R(1, \cdot)$.

(2) $S t_{R}^{\otimes}\left(\chi_{\{0\}}\right)(x)=\bigwedge_{z \in M}\left\{\chi_{\{0\}}(z) \rightarrow R(z, z \otimes x)\right\}=1 \rightarrow$ $R(0,0)=1$. Hence $S t_{R}^{\otimes}\left(\chi_{\{0\}}\right)=M$.

(3) $S t_{R}^{\otimes}(G)=\bigwedge_{z \in M}\{G(z) \rightarrow R(z, z \otimes x)\} \leq \bigwedge_{z \in M}\{F(z) \rightarrow$ $R(z, z \otimes x)\}=S t_{R}^{\otimes}(F)$,

$S t_{R}^{\oplus}(G)=\bigwedge_{z \in M}\{G(z) \rightarrow R(z, z \oplus \neg x)\} \leq \bigwedge_{z \in M}\{F(z) \rightarrow$ $R(z, z \oplus \neg x)\}=S t_{R}^{\oplus}(F)$.

(4) $S t_{R}^{\otimes}(F \cup G)=\bigwedge_{z \in M}\{F(x) \vee G(z) \rightarrow R(z, z \otimes x)\}$ $=\bigwedge_{z \in M}\{F(z) \rightarrow R(z, z \otimes x)\} \bigwedge_{z \in M}\{G(z) \rightarrow R(z, z \otimes x)\}$ $=S t_{R}^{\otimes}(F) \cap S t_{R}^{\otimes}(G)$,

$S t_{R}^{\oplus}(F \cup G)=\bigwedge_{z \in M}\{F(x) \vee G(z) \rightarrow R(z, z \oplus \neg x)\}=$ $\bigwedge_{z \in M}\{F(z) \rightarrow R(z, z \oplus \neg x)\} \bigwedge_{z \in M}\{G(z) \rightarrow R(z, z \oplus \neg x)\}$ $=S t_{R}^{\oplus}(F) \cap S t_{R}^{\oplus}(G)$.

(5) The proof of (5) is similar to (4).

(6) By Proposition 2.14, we have

$$
S t_{R}^{\oplus}(\widetilde{F})(x)=\bigwedge_{z \in M}\{\widetilde{F}(z) \rightarrow R(z, z \oplus \neg x)\}
$$




$$
\begin{aligned}
& =\bigwedge_{z \in M}\{F(\neg z) \rightarrow R(\neg z, \neg(z \oplus \neg x))\} \\
& \left.=\bigwedge_{z \in M}\{F(\neg z) \rightarrow R(\neg z, \neg z \otimes x))\right\} \\
& \left.=\bigwedge_{u=\neg z, z \in M}\{F(u) \rightarrow R(u, u \otimes x))\right\} \\
& \left.=\bigwedge_{u \in M}\{F(u) \rightarrow R(u, u \otimes x))\right\}=S t_{R}^{\otimes}(F)(x) .
\end{aligned}
$$

Theorem 3.5. Let $F$ be a fuzzy set of $M$. Then $S t_{R}^{\otimes}(F)$ and $S t_{R}^{\oplus}(F)$ are fuzzy filters of $M$.

Proof. (1) $S t_{R}^{\otimes}(F)(1)=\bigwedge_{z \in M}\{F(z) \rightarrow R(z, z \otimes 1)\}=$ $\bigwedge_{z \in M}\{F(z) \rightarrow R(z, z)\}=1 \geq S t_{R}^{\otimes}(F)(x)$ $z \in M$

(2) For $x \leq y$, by Propositions 2.11 and 2.12 we have

$$
\begin{aligned}
& S t_{R}^{\otimes}(F)(x)=\bigwedge_{z \in M}\{F(z) \rightarrow R(z, z \otimes x)\} \\
& =\bigwedge_{z \in M}\{F(z) \rightarrow R(z \leftrightarrow z \otimes x, 1)\} \\
& \leq \bigwedge_{z \in M}\{F(z) \rightarrow R(z \leftrightarrow z \otimes y, 1)\} \\
& \leq \bigwedge_{z \in M}\{F(z) \rightarrow R(z, z \otimes y)\}=S t_{R}^{\otimes}(F)(y) ;
\end{aligned}
$$

(3) $\forall x, y, z \in M$, we have

$$
\begin{aligned}
& (z \rightarrow z \otimes x) \otimes(z \rightarrow z \otimes y) \rightarrow(z \rightarrow z \otimes x \otimes y) \\
& =(z \rightarrow z \otimes x) \rightarrow[(z \rightarrow z \otimes y) \rightarrow(z \rightarrow z \otimes x \otimes y)] \\
& =(z \rightarrow z \otimes x) \rightarrow[z \otimes(z \rightarrow z \otimes y) \rightarrow z \otimes x \otimes y] \\
& =z \otimes(z \rightarrow z \otimes x) \otimes(z \rightarrow z \otimes y) \rightarrow z \otimes x \otimes y \\
& \geq z \otimes x \otimes(z \rightarrow z \otimes y) \rightarrow z \otimes x \otimes y \\
& \geq x \otimes z \otimes y \rightarrow z \otimes x \otimes y=1 .
\end{aligned}
$$

This means that $(z \rightarrow z \otimes x) \otimes(z \rightarrow z \otimes y)$ $\leq(z \rightarrow z \otimes x \otimes y)$. Then by Propositions 2.11 and 2.12 we have

$$
\begin{aligned}
S t_{R}^{\otimes}(F)(x \otimes y)=\bigwedge_{z \in M}\{F(z) \rightarrow R(z \leftrightarrow z \otimes x \otimes y, 1)\} \\
=\bigwedge_{z \in M}\{F(z) \rightarrow R(z \rightarrow z \otimes x \otimes y, 1)\} \\
\geq \bigwedge_{z \in M}\{F(z) \rightarrow R(z \rightarrow z \otimes x, 1) \wedge R(z \rightarrow z \otimes y, 1)\} \\
=\bigwedge_{z \in M}\{F(z) \rightarrow R(z \rightarrow z \otimes x, 1)\} \bigwedge_{z \in M}\{F(z) \rightarrow R(z \rightarrow z \otimes y, 1)\} \\
=\bigwedge_{z \in M}\{F(z) \rightarrow R(z, z \otimes x)\} \bigwedge_{\bigwedge_{z \in M}}\{F(z) \rightarrow R(z, z \otimes y)\} \\
=S t_{R}^{\otimes}(F)(x) \wedge S t_{R}^{\otimes}(F)(y) .
\end{aligned}
$$

Hence $S t_{R}^{\otimes}(F)$ is a fuzzy filter of $M$. The proof of that $S t_{R}^{\oplus}(F)$ is a fuzzy filter of $M$ is similar.

\section{Stabilizer of a fuzzy set with respect to another fuzzy set}

Definition 4.1. Let $F, G$ be two fuzzy sets of $M$. The $\vee$ stabilizer of $F$ with respect to $G$ is defined by:

$$
S t^{\vee}(F, G)(x)=\bigwedge_{z \in M}[F(z) \rightarrow G(z \vee x)]
$$

Example 4.2. The IMTL-algebra $M$ is defined as in Example 3.3. Given two fuzzy sets $F$ and $G$ of $M$ as follows:

$$
\begin{aligned}
& F(x)=\left\{\begin{array}{l}
0.9, \text { for } x=1, \\
0.8, \text { for } x \in\{b, c\}, \\
0.4, \text { for } x \in\{0, a, d\} .
\end{array}\right. \\
& G(x)=\left\{\begin{array}{l}
0.9, \text { for } x \in\{b, c, 1\}, \\
0.3, \text { for } x \in\{0, a, d\} .
\end{array}\right.
\end{aligned}
$$

Then the $\vee$-stabilizer of $F$ with respect to $G$ is

$$
S t^{\vee}(F, G)(x)= \begin{cases}1, & \text { for } x \in\{b, c, 1\}, \\ 0.9, & \text { for } x \in\{0, a, d\} .\end{cases}
$$

In the above computing, the implication operator $\rightarrow$ is taken as Lukasiewicz implication operator, i.e., $a \rightarrow b=$ $(1-a+b) \wedge 1$.

Theorem 4.3. (1) If $S t^{\vee}(F, G)=M$ then $F \subseteq G$. Conversely, if $F \subseteq G$ and $F$ is a fuzzy filter then $S t^{\vee}(F, G)=M$, particularly $S t^{\vee}(F, F)=M$;

(2) If $G$ is a fuzzy filter, then $G \subseteq S t^{\vee}(F, G)$;

(3) $S t^{\vee}\left(\chi_{\{0\}}, G\right)=G$;

(4) If $G$ is a fuzzy filter and $G(1)=1$, then $S t^{\vee}\left(\chi_{\{1\}}, G\right)=M$

(5) If $F_{1} \subseteq F_{2}$ and $G_{1} \subseteq G_{2}$, then $\operatorname{St}^{\vee}\left(F_{2}, G_{1}\right)$ $\subseteq S t^{\vee}\left(F_{1}, G_{2}\right)$;

(6) $S t^{\vee}\left(F, \bigcap_{i \in \Gamma} G_{i}\right)=\bigcap_{i \in \Gamma} S t^{\vee}\left(F, G_{i}\right)$;

(7) $S t^{\vee}\left(\bigcup_{i \in \Gamma} F_{i}, G\right)=\bigcap_{i \in \Gamma} S t^{\vee}\left(F_{i}, G\right)$.

Proof. (1) Since $S t^{\vee}(F, G)=M$, we have $\forall x \in M$,

$$
S t^{\vee}(F, G)(x)=\bigwedge_{z \in M}[F(z) \rightarrow G(z \vee x)]=1 .
$$

Hence $\forall z \in M, F(z) \rightarrow G(z \vee x)=1$, particularly, $F(x) \rightarrow$ $G(x \vee x)=F(x) \rightarrow G(x)=1$. So $F(x) \leq G(x)$ for all $x \in M$. That is $F \subseteq G$.

Conversely, let $F \subseteq G$. Then

$$
\begin{aligned}
& S t^{\vee}(F, G)(x)=\bigwedge_{z \in M}[F(z) \rightarrow G(z \vee x)] \\
& \geq \bigwedge_{z \in M}[F(z \vee x) \rightarrow G(z \vee x)]=1 .
\end{aligned}
$$

Hence $S t^{\vee}(F, G)=M$. 
(2) Since $\forall z \in M, F(z) \rightarrow G(z \vee x) \geq G(z \vee x) \geq G(x)$, we obtain

$$
S t^{\vee}(F, G)(x)=\bigwedge_{z \in M}[F(z) \rightarrow G(z \vee x)] \geq G(x)
$$

Therefore $G \subseteq S t^{\vee}(F, G)$.

(3) For any $x \in M, S t^{\vee}\left(\chi_{\{0\}}, G\right)(x)=\bigwedge_{z \in M}\left[\chi_{\{0\}}(z) \rightarrow\right.$ $G(z \vee x)]=G(x)$. Hence $S t^{\vee}\left(\chi_{\{0\}}, G\right)=G$.

(4) For any $x \in M, S t^{\vee}\left(\chi_{\{1\}}, G\right)(x)=\bigwedge_{z \in M}\left[\chi_{\{1\}}(z) \rightarrow\right.$ $G(z \vee x)]=1$. Hence $S t^{\vee}\left(\chi_{\{1\}}, G\right)=M$.

(5) For any $x \in M, S t^{\vee}\left(F_{2}, G_{1}\right)(x)=\bigwedge_{z \in M}\left[F_{2}(z) \rightarrow\right.$ $\left.G_{1}(z \vee x)\right] \leq \bigwedge_{z \in M}\left[F_{1}(z) \rightarrow G_{2}(z \vee x)\right]$. This means that $S t^{\vee}\left(F_{2}, G_{1}\right) \subseteq S t^{\vee}\left(F_{1}, G_{2}\right)$.

(6) For any $x \in M$,

$$
\begin{aligned}
& S t^{\vee}\left(F, \bigcap_{i \in \Gamma} G_{i}\right)(x)=\bigwedge_{z \in M}\left[F(z) \rightarrow \bigwedge_{i \in \Gamma} G_{i}(z \vee x)\right] \\
& =\bigwedge_{z \in M} \bigwedge_{i \in \Gamma}\left[F(z) \rightarrow G_{i}(z \vee x)\right] \\
& =\bigwedge_{i \in \Gamma} \bigwedge_{z \in M}\left[F(z) \rightarrow G_{i}(z \vee x)\right]=\bigwedge_{i \in \Gamma} S t^{\vee}\left(F, G_{i}\right)(x) .
\end{aligned}
$$

Therefore (6) holds.

(7) For any $x \in M$,

$$
\begin{aligned}
& S t^{\vee}\left(\bigcup_{i \in \Gamma} F_{i}, G\right)(x)=\bigwedge_{z \in M}\left[\bigvee_{i \in \Gamma} F_{i}(z) \rightarrow G(z \vee x)\right] \\
& =\bigwedge_{z \in M} \bigwedge_{i \in \Gamma}\left[F_{i}(z) \rightarrow G(z \vee x)\right] \\
& =\bigwedge_{i \in \Gamma} \bigwedge_{z \in M}\left[F_{i}(z) \rightarrow G(z \vee x)\right]=\bigwedge_{i \in \Gamma} S t^{\vee}\left(F_{i}, G\right)(x) .
\end{aligned}
$$

Therefore (7) holds.

Theorem 4.4. If $G$ is a fuzzy filter then $S t^{\vee}(F, G)$ is a fuzzy filter. If $G$ is also a fuzzy prime filter then $S t^{\vee}(F, G)$ is a fuzzy prime filter.

Proof. Suppose that $G$ is a fuzzy filter. Then

(1) $S t^{\vee}(F, G)(1)=\bigwedge_{z \in M}[F(z) \rightarrow G(z \vee 1)]$

$$
\begin{aligned}
& =\bigwedge_{z \in M}[F(z) \rightarrow G(1)] \geq \bigwedge_{z \in M}[F(z) \rightarrow G(z \vee x)] \\
& =S t^{\vee}(F, G)(x) .
\end{aligned}
$$

(2) Let $x \leq y$, we have

$$
\begin{aligned}
& S t^{\vee}(F, G)(x)=\bigwedge_{z \in M}[F(z) \rightarrow G(z \vee x)] \\
& \leq \bigwedge_{z \in M}[F(z) \rightarrow G(z \vee y)]=S t^{\vee}(F, G)(y)
\end{aligned}
$$

(3) $S t^{\vee}(F, G)(x \otimes y)=\bigwedge_{z \in M}[F(z) \rightarrow G(z \vee(x \otimes y))]$

$$
\begin{aligned}
& \geq \bigwedge_{z \in M}[F(z) \rightarrow G((z \vee x) \otimes(z \vee y))] \\
& \geq \bigwedge_{z \in M}[F(z) \rightarrow G(z \vee x) \wedge G(z \vee y)] \\
& =\bigwedge_{z \in M}[(F(z) \rightarrow G(z \vee x)) \wedge(F(z) \rightarrow G(z \vee y))] \\
& =\bigwedge_{z \in M}[F(z) \rightarrow G(z \vee x)] \bigwedge \bigwedge_{z \in M}[F(z) \rightarrow G(z \vee y)] \\
& =S t^{\vee}(F, G)(x) \wedge S t^{\vee}(F, G)(y)
\end{aligned}
$$

This shows that $S t^{\vee}(F, G)$ is a fuzzy filter.

If $G$ is also a fuzzy prime filter, then

$$
\text { (4) } \begin{aligned}
& S t^{\vee}(F, G)(x \vee y)=\bigwedge_{z \in M}[F(z) \rightarrow G(z \vee x \vee y)] \\
= & \bigwedge_{z \in M}[F(z) \rightarrow G((z \vee x) \vee(z \vee y))] \\
\geq & \bigwedge_{z \in M}[F(z) \rightarrow G(z \vee x) \vee G(z \vee y)] \\
= & \bigwedge_{z \in M}[(F(x) \rightarrow G(z \vee x)) \vee(F(x) \rightarrow G(z \vee y))] \\
\geq & \bigwedge_{z \in M}[F(z) \rightarrow G(z \vee x)] \bigvee \bigwedge_{z \in M}[F(z) \rightarrow G(z \vee y)] \\
= & S t^{\vee}(F, G)(x) \vee S t^{\vee}(F, G)(y) .
\end{aligned}
$$

This shows that $S t^{\vee}(F, G)$ is a fuzzy prime filter.

Definition 4.5. Let $F, G$ be two fuzzy filters. For fuzzy set $F \otimes G:(F \otimes G)(x)=F(x) \otimes G(x), \forall x \in M$, the generated fuzzy filter of $F \otimes G$ is denoted by $F \widetilde{\otimes} G$, i.e., $\forall x \in M$,

$$
(F \widetilde{\otimes} G)(x)=\bigvee\left\{\bigwedge_{i=1}^{m}\left(F\left(a_{i}\right) \otimes G\left(a_{i}\right)\right) \mid x \geq a_{1} \otimes \cdots \otimes a_{m}\right\} .
$$

Theorem 4.6. Let $F, G, H$ be three fuzzy filters of $M$. Then $F \widetilde{\otimes} G \subseteq H$ if and only if $F \subseteq S t^{\vee}(G, H)$.

Proof. Suppose that $F \widetilde{\otimes} G \subseteq H$. Then $\forall x \in M, F(x) \otimes$ $G(x) \leq(F \widetilde{\otimes} G)(x) \leq H(x)$. Hence

$$
\begin{aligned}
& S t^{\vee}(G, H)(x)=\bigwedge_{z \in M}[G(z) \rightarrow H(z \vee x)] \\
& \geq \bigwedge_{z \in M}[G(z \vee x) \rightarrow H(z \vee x)] \\
& \geq \bigwedge_{z \in M}[G(z \vee x) \rightarrow F(z \vee x) \otimes G(z \vee x)] \\
& \geq \bigwedge_{z \in M} F(z \vee x) \geq F(x) .
\end{aligned}
$$

This means that $F \subseteq S t^{\vee}(G, H)$. 
Conversely, suppose that $F \subseteq S t^{\vee}(G, H)$. Then $\forall x \in$ $M, F(x) \leq S t^{\vee}(G, H)(x)=\bigwedge_{z \in M}[G(z) \rightarrow H(z \vee x)]$. Hence $F(x) \leq G(x) \rightarrow H(x \vee x)=G(x) \rightarrow H(x)$. It follows that $F(x) \otimes G(x) \leq H(x)$ for all $x \in M$. Thus

$$
\begin{aligned}
& (F \widetilde{\otimes} G)(x)=\bigvee\left\{\bigwedge_{i=1}^{m}\left(F\left(a_{i}\right) \otimes G\left(a_{i}\right)\right) \mid x \geq a_{1} \otimes \cdots \otimes a_{m}\right\} \\
& \leq \bigvee\left\{\bigwedge_{i=1}^{m}\left(H\left(a_{i}\right) \mid x \geq a_{1} \otimes \cdots \otimes a_{m}\right\}\right. \\
& =\bigvee\left\{\left(H\left(a_{1} \otimes \cdots \otimes a_{m}\right) \mid x \geq a_{1} \otimes \cdots \otimes a_{m}\right\}\right. \\
& \leq H(x) .
\end{aligned}
$$

This shows that $F \widetilde{\otimes} G \subseteq H$.

On the set $\mathscr{F}(M)$, if we denote $F \leq G$ if and only if $F \subseteq G, F \wedge G=F \cap G, F \vee G=\langle F \cup G\rangle$, then $(\mathscr{F}(M), \leq, \wedge, \vee)$ is a bounded distributive lattice (see [19]). By Theorem 4.6, we have the following conclusion.

Theorem 4.7. $\left(\mathscr{F}(M), \leq, \wedge, \vee, \widetilde{\otimes}, S t^{\vee}(\cdot, \cdot)\right)$ is a residuated lattice.

\section{Conclusion}

In this paper, we introduced two types of fuzzy stabilizers $S t_{R}^{\otimes}(F)$ and $S t_{R}^{\oplus}(F)$ of a fuzzy set $F$ and the stabilizer $S t^{\vee}(F, G)$ of $F$ with respect to $G$ in an IMTL-algebra. We investigated some properties of the stabilizers and shows that $S t_{R}^{\otimes}(F), S t_{R}^{\oplus}(F)$ and $S t^{\vee}(F, G)$ are fuzzy filters of $M$. By introducing the $\widetilde{\otimes}$ operation of two fuzzy filters, we prove that $\widetilde{\otimes}$ and $S t^{\vee}(\cdot, \cdot)$ form an adjoint pair and the set of all fuzzy filters $\mathscr{F}(M)$ is a residuated lattice.

\section{Acknowledgement}

The authors are grateful to the anonymous referee for a careful checking of the details and for helpful comments that improved this paper.

\section{References}

[1] G. Görz, S. Hölldobler, Advances in Artificial Intelligence, Lecture Notes in Artificial Intelligence, Springer-Verlag, New York, (1996).

[2] C.Y. Shi, C.N. Huang, J.Q. Wang, Principle of Artificial Intelligence, Tsinghua University Press, Beijing, (1993).

[3] P. Hájek, Metamathematics of Fuzzy Logic, Kluwer Adacemic Publishers, Dordrecht, (1998).

[4] F. Esteva, L. Godo, Monoidal $t$-norm based logic: towards a logic for left-continuous $t$-norms, Fuzzy Sets and Systems, 124, 271-288 (2001).

[5] G.J. Wang, A formal deductive system for fuzzy reasoning calculus, Chinese Science Bulletin, 42, 1521-1526 (1997).
[6] G.J. Wang, G.J. Wang, Non-classical mathematical logic and approximate reasoning, Beijing: Science Press, (2000).

[7] G.J. Wang, MV-algebras, BL-algebras, IMTL-algebras and mutiple-valued logic, Fuzzy Systems and Mathematics, 16, 1-15 (2002).

[8] D.W. Pei, G.J. Wang, The completeness and applications of the formal system $\mathscr{L}^{*}$, Science in China(Series E), 32, 56-64 (2002).

[9] D.W. Pei, On equivalent forms of fuzzy logic systems NM and IMTL, Fuzzy Sets and Systems, 138, 187-195 (2003).

[10] E. Turunen, Mathematics Behind Fuzzy Logic, PhysicaVerlag, Heidelberg, (1999).

[11] E. Turunen, BL-algebras and basic fuzzy logic, Mathware and Soft Computing, 6, 49-61 (1999).

[12] E. Turunen, Boolean deductive systems of BL-algebras, Archive Mathematical Logic, 40, 467-473 (2001).

[13] Y. Xu, Lattice impliation algebras, J. Southwest Jiaotong University, 1, 20-27 (1993).

[14] Y. Xu, K.Y. Qin, On filters of lattice implication algebras, J. Fuzzy Mathematics, 1, 251-260 (1993).

[15] Y.L. Liu, S.Y. Liu, Y. Xu, K.Y. Qin, ILI-ideals and prime LI-ideals in lattice impliation algebras, Information Sciences, 155, 157-175 (2003).

[16] Y.B. Jun, Fuzzy positive implicative and fuzzy associative filters of lattice impliation algebras, Fuzzy Sets and Systems, 121, 353-357 (2001).

[17] L.Z. Liu, K.T. Li, Fuzzy implicative and Boolean filters of $R_{0}$-algebras, Information Sciences, 171, 61-71 (2005).

[18] L.Z. Liu, K.T. Li, Fuzzy Boolean and positive implicative filters of BL-algebras, Fuzzy Sets and Systems, 152, 333-348 (2005).

[19] J.L. Zhang, H.J. Zhou, Fuzzy filters on the residuated lattices, New Mathematics and Natural Computation, 2, 1128 (2006).

[20] R. Balbes, P. Dwinger, Distributive Lattice, University of Missouri Press, Columbia, (1974).

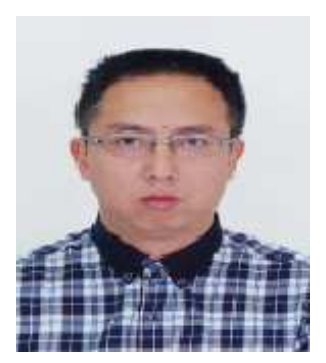

Maosen Xie was born in 1968 in Sichuan, P.R. China. He received his B.Sc. (1990) in Mathematics from the Chongqing Normal University and M.Sc. (2007) in Mathematics from the University of Electronic Science and Technology, P.R. China. His research interests include approximate reasoning, fuzzy set and random set. $\mathrm{He}$ is the author and coauthor of more than 10 academic journal papers and research monographs 